\title{
Total Variation regularization enhances regression-based brain activity prediction
}

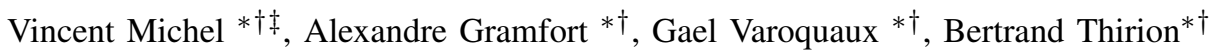 \\ *Parietal team, INRIA Saclay-Ile-de-France, Saclay, France \\ $\dagger$ CEA, DSV, I2BM, NeuroSpin, Saclay, France \\ $\ddagger$ Université Paris-Sud 11, Orsay, France
}

\begin{abstract}
While medical imaging typically provides massive amounts of data, the automatic extraction of relevant information in a given applicative context remains a difficult challenge in general. With functional MRI (fMRI), the data provide an indirect measurement of brain activity, that can be related to behavioral information. It is now standard to formulate this relation as a machine learning problem where the signal from the entire brain is used to predict a target, typically a behavioral variable. In order to cope with the high dimensionality of the data, the learning method requires a regularization procedure. Among other alternatives, $\ell_{1}$ regularization achieves simultaneously a selection of the most predictive features. One limitation of the latter method, also referred to as Lasso in the case of regression, is that the spatial structure of the image is not taken into account, so that the extracted features are often hard to interpret. To obtain more informative and interpretable results, we propose to use the $\ell_{1}$ norm of the image gradient, a.k.a., the Total Variation (TV), as regularization. TV extracts few predictive regions with piecewise constant weights over the whole brain, and is thus more consistent with traditional brain mapping. We show on real fMRI data that this method yields more accurate predictions in inter-subject analysis compared to voxel-based reference methods, such as Elastic net or Support Vector Regression.
\end{abstract}

Keywords-fMRI; regression; regularization; Total Variation; spatial structure

\section{INTRODUCTION}

Inferring behavioral information or cognitive states from brain activation images such as those obtained with functional magnetic resonance imaging (fMRI) is a recent neuroimaging data analysis paradigm ? that can provide more sensitive analyzes than standard statistical parametric mapping procedures ?. This approach can be used to assess the involvement of one or several brain regions in certain cognitive or perceptual functions, by evaluating the accuracy of the prediction of a behavioral variable of interest (the target). This inference generally uses a prediction function whose accuracy depends on its ability to use the relevant variables, i.e., the correct brain regions. Importantly, inference methods should simultaneously lead to good prediction performance and provide an interpretable model: the predictive function learned from the data should be as explicit as standard statistical mapping results. This objective is addressed by the TV regression presented in this contribution.

Many methods have been tested for classification or regression of fMRI activation images (Linear Discriminant Analysis, Support/Relevance Vector Machines, Lasso, Elastic net regression and many others), but in this problem the major bottleneck remains the extraction of predictive information within the brain volume (see ? for a review). In practice, feature selection is important to achieve accurate prediction: when the number of features (voxels) is much larger than the numbers of samples (images), the prediction method overfits the training set, and thus does not generalize well. Besides, feature selection drastically reduces the spatial support of predictive regions, and thus potentially provides a simpler spatial distribution of the predictive features than whole-brain maps.

To date, the most widely used method for feature selection is voxel-based Anova (Analysis of Variance), that evaluates each voxel independently. This is often combined with a Support Vector Machines approach as prediction function. However, it is suboptimal to perform feature selection and parameter estimation procedures separately, and there is a lot of interest in regularization methods that perform both simultaneously.

Let us introduce the following regression model:

$$
y=X w+\epsilon
$$

where $y$ represents the target data $\left(y \in \mathbb{R}^{n}\right)$ and $w$ the parameters to be estimated. The vector $w \in \mathbb{R}^{m}$ can be seen as an image; $m$ is the number of features (or voxels). The matrix $X \in \mathbb{R}^{n \times m}$ is the design matrix. Each row is an $m$ dimensional sample. The crucial issue here is that $n \ll m$, so that estimating $w$ is an ill-posed problem. The estimation requires therefore adapted regularization.

A standard approach to perform the estimation of $w$ with regularization uses penalization of the maximum likelihood estimator. This leads to the following minimization problem:

$$
\hat{w}=\arg \min _{w}\|y-X w\|^{2}+J(w)
$$

where $J(w)$ is the penalization term.

The reference method is Elastic net (see ?), which is a combined $\ell_{1}$ and $\ell_{2}$ penalization $J(w)=\lambda_{1}\|w\|_{1}^{1}+\lambda_{2}\|w\|_{2}^{2}$. Elastic net has two limit cases: $\lambda_{2}=0$ is the Lasso ? which yields an extreme sparsity in the selected features, and $\lambda_{1}=0$ corresponds to Ridge regression. 
However, such a penalization does not take into account the underlying structure of $w$, i.e., a spatial 3-dimensional grid in the case of brain images. The main motivation for using this spatial structure is that the predictive information is organized in regions, and not randomly spread across voxels. In this article, we develop an approach for regularized regression based on Total Variation (TV), that we call $T V$ regression. TV ends up providing an estimate $\hat{w}$ of $w$ with a sparse block structure, from which the regions involved in the cognitive task can be extracted.

Mathematically TV, which has been widely used in image denoising ?, is defined as the $\ell_{1}$ norm of the gradient of the image:

$$
T V(w)=\int_{\omega \in \Omega} \sqrt{\nabla_{x} w(\omega)^{2}+\nabla_{y} w(\omega)^{2}+\nabla_{z} w(\omega)^{2}} d \omega
$$

In this contribution, the mathematical and implementation details of $T V$ regression are first detailed. It is then applied to an fMRI paradigm that studies object size characterization. Results show that TV outperforms other reference methods, as it yields better prediction performance while providing weights $\hat{w}$ with an interpretable spatial structure.

\section{Methods}

\section{A. Total Variation regression}

The computational procedure used for $T V$ regression is based on the gradients of the $\ell_{2}$ data fidelity term in Eq. (2) and the computation of the proximity operator associated with the TV penalty.

Definition (Proximity operator). Let $J: \mathbb{R}^{m} \rightarrow \mathbb{R}$ be a lower semi-continuous, convex function. The proximity operator associated with $J$ and $\lambda \in \mathbb{R}_{+}$denoted by $\operatorname{prox}_{\lambda J}: \mathbb{R}^{m} \rightarrow \mathbb{R}^{m}$ is given by

$$
\operatorname{prox}_{\lambda J}(w)=\arg \min _{v \in \mathbb{R}^{m}} \frac{1}{2}\|v-w\|_{2}^{2}+\lambda J(v) .
$$

In the particular case of $\mathrm{TV}$, the proximity operator is known as the ROF problem ? and recent results ? have shown that it could be solved efficiently with first order iterative procedures. The pseudo code is provided in Algorithm 1. For details and proof of convergence of the algorithm see ?. In practice, the stopping condition for the iterative computation of the TV proximity operator is based on the computation of a duality gap. This guarantees the optimality of the solution (it sets the $P$ variable). The number of iterations $N$ is fixed to 500 as it turns out to lead to an acceptable convergence using the fMRI data presented here. A difficulty specific to fMRI data is the computation of the gradient and divergence over a mask of the brain with correct border conditions. Moreover, with such an irregular domain, the Lipschitz constant $L$ also needs to be estimated on each input data. To do this we use a power method classically used to estimate the spectral norm of a linear operator.

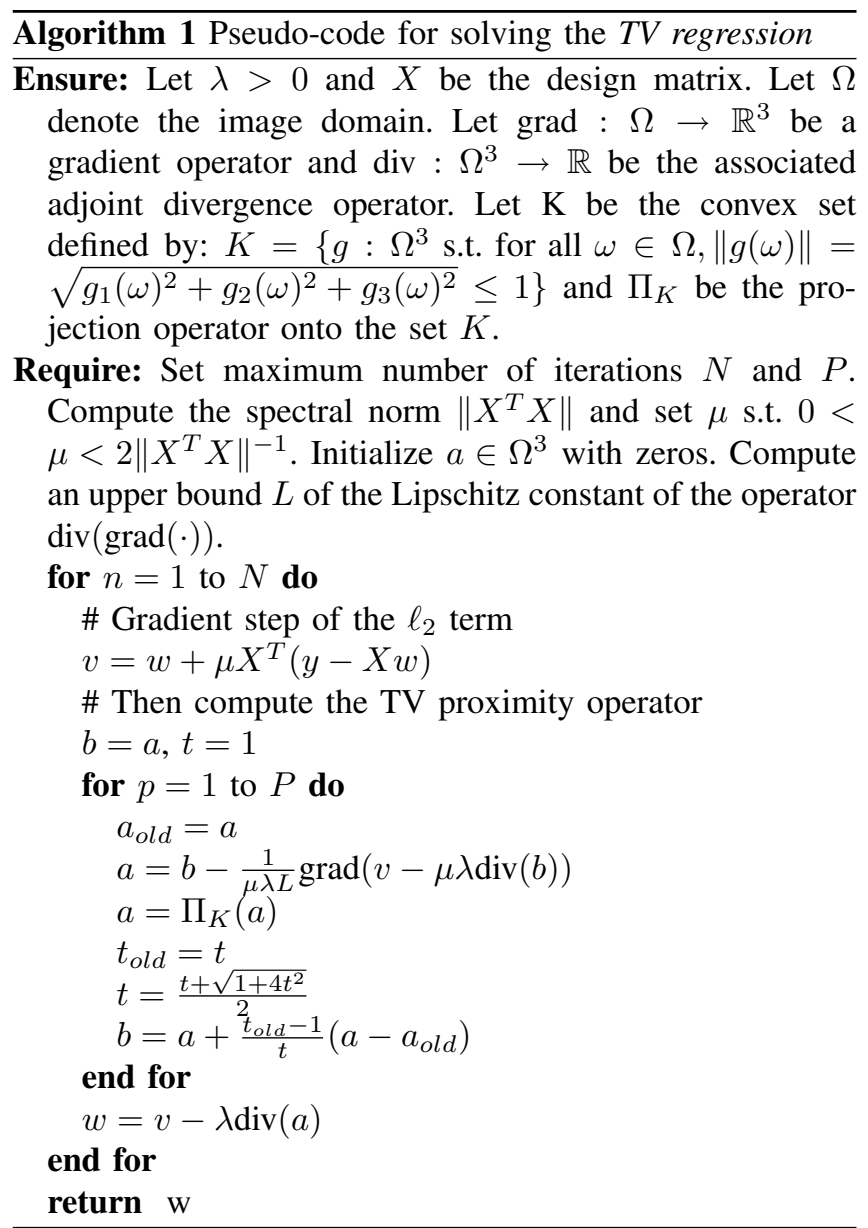

\section{B. Performance evaluation}

Our method is evaluated with a cross-validation procedure that splits the available data into training and validation sets (here we use a leave-one-subject-out procedure). In the following, $\left(X^{l}, y^{l}\right)$ are a learning set, $\left(X^{t}, y^{t}\right)$ a test set and $\hat{y}^{t}=X^{t} \hat{w}$ refers to the predicted target, where $\hat{w}$ is estimated from the training set. The performance of the different regression models is evaluated using $\zeta$, the ratio of explained variance (or $R^{2}$ coefficient):

$$
\zeta\left(X^{l}, y^{l}, X^{t}, y^{t}\right)=\frac{\operatorname{var}\left(y^{t}\right)-\operatorname{var}\left(y^{t}-\hat{y}^{t}\right)}{\operatorname{var}\left(y^{t}\right)}
$$

This is the amount of variability in the response that can be explained by the model (perfect prediction yields $\zeta=1$, while $\zeta<0$ if prediction is worse than chance).

\section{Competing methods}

The $T V$ regression is compared to different reference methods :

- Elastic Net regression, that requires a double optimization, for the two parameters $\lambda_{1}$ and $\lambda_{2}$. A crossvalidation procedure within the training set is used to optimize these parameters. Here, we use $\lambda_{1} \in$ 
$\{0.2 \tilde{\lambda}, 0.1 \tilde{\lambda}, 0.05 \tilde{\lambda}, 0.01 \tilde{\lambda}\}$, where $\tilde{\lambda}=\left\|X^{T} y\right\|_{\infty}$, and $\lambda_{2} \in\{0.1,0.5,1 ., 10 ., 100$.$\} .$

- Support Vector Regression ( SVR) with a linear kernel (see ?), which is the reference method in neuroimaging, due to its robustness in large dimension. The $C$ parameter is optimized by cross-validation in the range $10^{-3}$ to $10^{1}$ in multiplicative steps of 10 .

Both of these methods are used jointly after an Anova-based feature selection as this maximizes their performance. This selection is performed on the training set of each fold in the cross-validation loop, and the optimal number of voxels is selected within the range $\{50,100,250,500\}$. The three methods are developed in Python. Both Elastic Net and SVR are freely available in the Scikit-learn package ?.

\section{EXPERIMENTS AND RESULTS}

\section{A. Experiments on Real Data}

We used a real fMRI dataset related to an experiment on the representation of objects, as detailed in ?. During the experiment, ten healthy volunteers viewed objects of three different sizes and four different shapes, with 4 repetitions of each stimulus in each one of 6 sessions. Functional images were acquired on a 3-T MR system with eightchannel head coil (Siemens Trio, Erlangen, Germany) as T2*-weighted echo-planar image (EPI) volumes. Twenty transverse slices were obtained with a repetition time of $2 \mathrm{~s}$ (echo time, $30 \mathrm{~ms}$; flip angle, $70^{\circ} ; 2 \times 2 \times 2$-mm voxels; 0.5-mm gap). Realignment, normalization to MNI space, and General Linear Model (GLM) fit were performed with the SPM5 software. In the present work we used the resulting session-wise parameter estimate images. The four different shapes of objects are pooled across the three sizes, and we are interested in discrimination between sizes. This can be handled as a regression problem, where we aim at predicting the size of an object corresponding to a new fMRI scan.

We perform an inter-subjects analysis that relies on subjectspecific fixed-effects activations (across repetitions). This yields a total of 12 images by subject, with 4 images for each 3 sizes of object. Thus, the dimensions of the real data set are $m \sim 7 \times 10^{4}$ and $n=120$ (divided in 3 sizes). We evaluate the performance of the method by cross-validation (leaveone-subject-out), which yields an average rate of explained variance across subjects. This analysis is launched on the whole brain volume.

The parameters of the reference methods are optimized with a leave-one-subject-out cross-validation within the training set, using a three-way grid search in the ranges given before. In the $T V$ regression procedure, the $\lambda$ parameter is set via the definition of an auxiliary variable $\alpha=\lambda / n$ where $n$ is the number of images. This scaling makes the setting of the regularization parameter easier and more stable between different datasets.

\section{B. Results on Real Data}

The results found by the three methods are given in Table I. TV regression outperforms the two alternative methods, yielding an average explained variance of $81 \%$. Moreover, the predictions of $T V$ regression are more stable than the ones of the two reference methods, with a standard deviation of the explained variance two times smaller than the $S V R$. The weight maps found for different values of the regularization parameter $\lambda$ are shown in Fig.1. It can be seen that, when $\lambda$ increases, the spatial support of these maps tends to be aggregated in very few clusters within the occipital cortex, and that they have a nearly constant value on these clusters. When $\lambda$ decreases, the $T V$ regression algorithm is able to create small informative clusters within the occipital cortex, that are comparable to standard activation maps, but where most of the brain regions are shrunk toward 0 . By contrast, both reference methods yield uninterpretable maps, with a few informative voxels spread out in the whole occipital cortex, so that it is very difficult to identify meaningful brain structures from these maps.

Table I

SCORES OF EXPLAINED VARIANCE FOR THE DIFFERENT METHODS

\begin{tabular}{|l|c|c|c|c|c|}
\hline Methods & $\operatorname{mean} \zeta$ & $\operatorname{std} \zeta$ & $\max \zeta$ & $\min \zeta$ & Time (s) \\
\hline \hline SVR & 0.7 & 0.17 & 0.92 & 0.4 & 151 \\
Elastic net & 0.75 & 0.14 & 0.96 & 0.48 & 2428 \\
TV & 0.81 & 0.08 & 0.97 & 0.7 & 241 \\
\hline
\end{tabular}

All three methods have also been tested in an intra-subject analysis using the same dataset. In that case, they lead to very similar results in terms of performance, although the $S V R$ yields slightly better accuracy than $T V$ regression. This is due to the fact that the voxel-to-voxel correspondence between images is valid in an intra-subject analysis compared to an inter-subject analysis. However, the voxel-based approaches still suffer from the limitation that the maps obtained are very hard to interpret.

\section{DISCUSSION}

Regularization of voxels loadings significantly increases the generalization ability in regression problems. However, regularization is commonly performed without using the spatial structure of the images. The proposed approach performs an adaptive and efficient regularization, while creating interpretable weighted maps with regions of constant weights. Thus, the $T V$ regression method fulfills the two requirements that make it suitable for neuroimaging: a good prediction accuracy (equal to or better than the reference methods), and a set of interpretable features, i.e., clusters of similarly tuned voxels. Especially, in the case of a multi-subject study, considering extended regions is expected to compensate for spatial misalignment between individual datasets, hence can better generalize than voxel-based methods. Another asset of the $T V$ regression is that it allows to consider the whole brain in the analysis, without requiring any prior feature selection. Finally, an important feature of our implementation is that it 
reduces computation time to a reasonable amount, so that it is not significantly more costly than SVR or Elastic Net in practical settings (i.e., including the cross-validation loops, see Table I).

From a neuroscientific point of view, the selected regions from a whole brain analysis are concentrated in the early visual cortex. This is consistent with the fact that early visual cortex yields highly reliable signals that are discriminative of feature/shape differences between object exemplars, which holds as long as no high-level generalization across images is required (see e.g. ? and ?). Finally, the spatial pattern of this information is stable enough across subjects to be extracted and used to make reliable predictions.

\section{CONCLUSION}

In this paper we proposed to adapt $T V$ regression for extracting information from brain images. The feature selection and model estimation are performed jointly and capture the predictive information present in the data better than alternative methods. A particularly important property of this approach is its ability to create spatially coherent regions with similar weights, yielding interpretable and still informative sets of features. Experimental results show that this algorithm performs particularly well on real data in a multi-subject analysis. These observations demonstrate that $T V$ regression is a powerful tool for understanding brain activity.

Acknowledgements: The authors acknowledge support from the ANR grant ViMAGINE ANR-08-BLAN-0250-02.
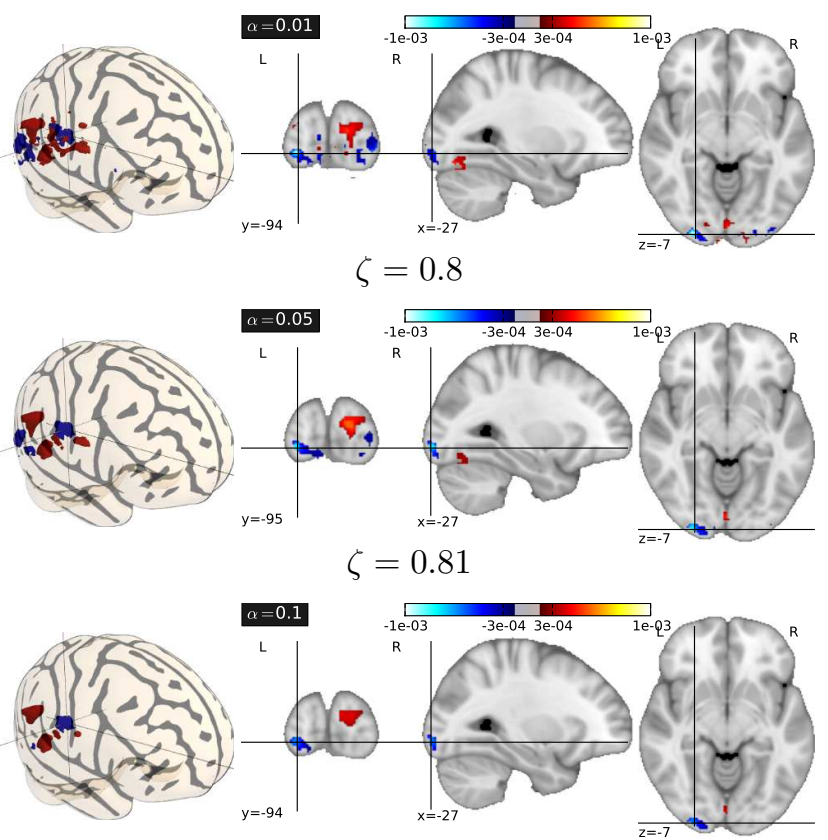

$$
\zeta=0.81
$$

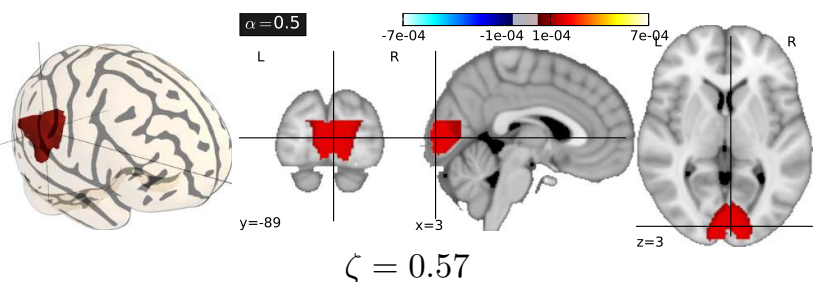

Figure 1. Maps of weights found by $T V$ regression for different values of the regularization parameter $\alpha$. When it decreases, the $T V$ regression algorithm creates different clusters of weights with constant values. These clusters are more easily interpretable than voxel-based map (see bellow). Moreover, the clusters are found in the visual cortex, as expected, and show an interesting spatial structure which seems symmetrical: clusters with negative weights are more lateral than clusters with positive weights, and less ventral. The $T V$ regression algorithm is stable for different values of $\alpha$ in the range $[0.01,0.1]$, has shown by the explained variance $\zeta$.
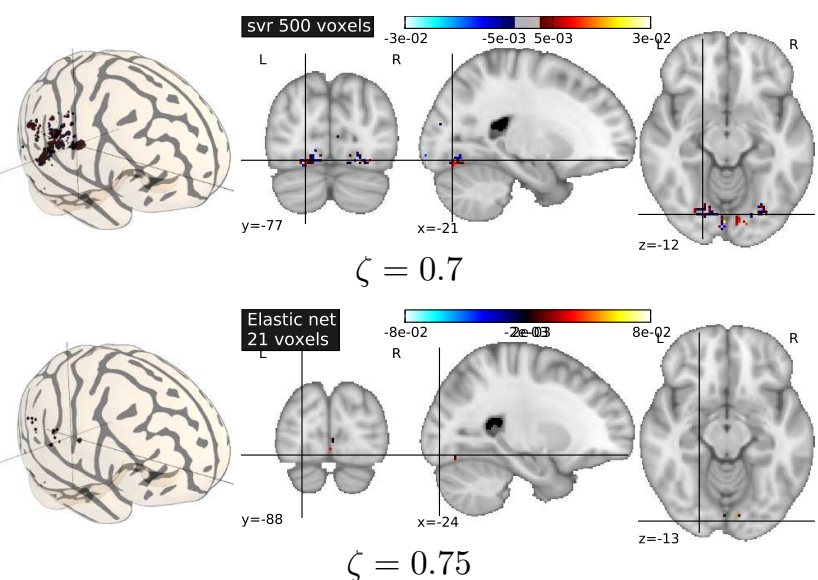

Figure 2. Maps of weights found by the SVR (up) and Elastic net (bottom) methods. The optimal number of voxels selected by Anova is 500, but Elastic net further reduces this set to 21 voxels. These voxel-based methods yield features that are difficult to interpret (especially when compared to $T V$ regression ), which is related to the fact that they do not consider the spatial structure of the image 